\title{
Is there any potential link among caspase-8, p-p38 MAPK and bcl-2 in clear cell renal cell carcinomas? A comparative immunohistochemical analysis with clinical connotations
}

\author{
Vassilis Samaras*1, Maria Tsopanomichalou ${ }^{\dagger 1}$, Angeliki Stamatelli ${ }^{\dagger 2}$, \\ Christos Arnaoutoglou ${ }^{3}$, Efstathios Samaras ${ }^{4}$, Marianthi Arnaoutoglou ${ }^{5}$, \\ Hercules Poulias ${ }^{6}$ and Calypso Barbatis ${ }^{1}$
}

Address: ${ }^{1}$ Department of Pathology, Hellenic Red Cross Hospital, Athens, Greece, ${ }^{2} 1$ st Department of Pathology, University of Athens Medical School, Athens, Greece, ${ }^{3}$ Department of Cytopathology, Evangelismos General Hospital, Athens, Greece, ${ }^{4}$ Department of Neurosurgery, Hellenic Red Cross Hospital, Athens, Greece, ${ }^{5} 1$ st Department of Neurology, Aristotle University of Thessaloniki, Thessaloniki, Greece and ${ }^{6}$ Department of Urology, Hellenic Red Cross Hospital, Athens, Greece

Email:Vassilis Samaras* - vassilissamaras@yahoo.gr; Maria Tsopanomichalou - voulaki24@yahoo.gr; Angeliki Stamatelli - agstam@med.uoa.gr; Christos Arnaoutoglou - arnaoutoglou@inbox.com; Efstathios Samaras - stathissamaras@gmail.com; Marianthi Arnaoutoglou - arnaoutoglou@inbox.com; Hercules Poulias - vassilissamaras@gmail.com;

Calypso Barbatis - vassilissamaras@gmail.com

* Corresponding author †Equal contributors

Published: 17 February 2009

Diagnostic Pathology 2009, 4:7 doi:10.1186/1746-1596-4-7

This article is available from: http://www.diagnosticpathology.org/content/4/I/7

(C) 2009 Samaras et al; licensee BioMed Central Ltd.

This is an Open Access article distributed under the terms of the Creative Commons Attribution License (http://creativecommons.org/licenses/by/2.0), which permits unrestricted use, distribution, and reproduction in any medium, provided the original work is properly cited.

\begin{abstract}
Background: Clear cell renal cell carcinomas (ccRCCs) constitute the most common renal carcinomas, characterized by a relatively aggressive clinical course. Thus, scientific research is targeting towards the identification of immunohistochemical and molecular markers that could be useful regarding diagnosis, appropriate therapy and prediction of prognosis. In the present study we assessed and correlated the expression of caspase- 8 , phosphorylated p38 mitogen-activated protein kinase ( $\mathrm{p}-\mathrm{p} 38)$ and bcl-2 protein with histopathological features and clinical outcome of 27 patients with ccRCCs.
\end{abstract}

Method: Immunohistochemistry in formalin-fixed and paraffin-embedded tissue sections was performed. The associations among various features were assessed utilizing statistical analysis.

Results: We found that increased expression of cytoplasmic caspase-8 and bcl-2 protein was strongly associated with low Fuhrman's grade of carcinomas $(p=0.019$ and $p=0.04 \mathrm{I}$, respectively). On the other hand, increased $p-p 38$ expression was significantly related to high Fuhrman's grade $(p=0.006)$. Moreover, high bcl-2 expression was correlated with low pathological stage of $\operatorname{ccRCCs}(p=0.026)$. Increased expression of cytoplasmic caspase-8 as well as low-grade tumors (grade I and 2) implied a greater probability of patients' survival, in univariate statistical analysis $(p=0.037$ and $p=0.019$, respectively). Neither p-p38 nor bcl-2 expression was significantly linked to patients' survival. There were not emerged statistically significant associations among caspase-8, p-p38 kinase and bcl-2 protein.

Conclusion: For the first time the prognostic impact of caspase-8 and p-p38 was studied in a series of ccRCCs, using immunohistochemistry in formalin-fixed and paraffin-embedded tissue sections. The suggestive relationship of caspase- 8 with patients' clinical outcome, as well as the role of p-p38 within different grade categories, mandates further studies in larger cohorts of RCCs. 


\section{Background}

Conventional (clear) RCCs (ccRCCs) are characterized by their relatively poor prognosis due to late presentation and resistance to various therapeutical manipulations [1]. Thus, the identification of new immunohistochemical and molecular markers that could provide better prognostic information for each patient is needed. In this context, the role of factors, such as bcl-2 protein, caspases and mitogen-activated protein kinases (MAPKs), influencing cell survival-proliferation and apoptosis, has not been fully understood yet, within ccRCCs.

Bcl-2 is an intracellular membrane protein capable of blocking the programmed cell death through regulation of the mitochondrial apoptotic pathway $[2,3]$. Although bcl-2 is frequently upregulated in RCCs, studies of its exact relationship with patients' clinicopathological features have given controversial results thus far [4-6].

Caspases are proteases characterized by their essential action in the apoptotic pathway [7]. Caspase-8, particularly, plays a decisive role as an initiator caspase in the extrinsic apoptotic pathway since its activation leads to caspase-3 activation and accordingly to apoptosis [7]. However, bibliographical data regarding caspase-8 immunohistochemical expression in RCCs are limited [8-10].

p38, a member of the MAPKs group, participates in a signaling cascade regulating cellular responses to cytokines and stress and seems to play a role in cell proliferation and apoptosis within RCCs $[11,12]$. Few former studies assessed the immunohistochemical expression of p38 in RCCs but the impact of activated (phosphorylated) p38 (p-p38) on patients' survival has not been described yet $[12,13]$.

Experimental data suggest that $\mathrm{p} 38$ pathway may function both upstream and downstream of caspases (including caspase-8) in the apoptotic response $[14,15]$. Moreover, p38 kinase appears to play a crucial role regarding bcl-2 phosphorylation and its subsequent inactivation, leading to an increased apoptotic effect $[16,17]$. Nevertheless, this dual, yet controversial, function of p38 kinase which links three different apoptotic pathways has never been studied within RCCs.

The purpose of our study was to assess and compare the immunohistochemical expression of p-p38, bcl-2 and caspase- 8 in ccRCCs, identifying any associations with Fuhrman's grade, pathological stage and patients' clinical outcome. To our knowledge this is the first study attempting to explore the specific prognostic influence of p-p38 and caspase- 8 immunohistochemical expression within ccRCCs.

\section{Method}

27 patients with ccRCCs [ 15 men and 12 women, mean age at operation: 58.15 years (range: $42-77$ )] underwent radical nephrectomy between 1999 and 2003 at our Hospital. Follow-up information was available in all cases, informed consent was obtained from all patients and the study was approved by the scientific committee of the Hospital. The mean time of follow-up was 70.63 months (range: 11-105), during which 4 patients died of the disease [mean survival: 40.75 months (range: 11-54)] whereas 23 were alive [mean follow-up: 75.83 months (range: 44-105)].

All specimens were formalin-fixed and paraffin-embedded. For each patient, one representative block containing more than $80 \%$ of neoplastic tissue was histopathologically evaluated. All tumors were graded according to Fuhrman's grading system [18] as: grade $1(n=7)$, grade $2(n$ $=13)$, grade $3(n=4)$ and grade $4(n=3)$. The pathological stage (pT-stage) was determined using the TNM classification of malignant tumors [19] as: pT1a $(n=5)$, pT1b $(n=6)$, pT2 $(n=8)$, pT3a $(n=5)$ and pT3b $(n=3)$.

p-p38, bcl-2 and caspase-8 immunostaing was performed in representative sections, cut at $3 \mu \mathrm{m}$, using Bond-maX system (Vision BioSystems Ltd, Australia) according to the manufacturer's instructions. The following antibodies were used: anti-phopsho-p38 MAPK (clone 12F8, rabbit $\mathrm{mAb}$, Cell Signaling Technology Inc. USA, diluted: 1/ 100 ), anti-bcl-2 protein (clone 124, mouse mAb, Dako Cytomation, Denmark, diluted: 1/50) and anti-caspase- 8 (clone 11B6, mouse mAb, Novocastra, UK, diluted: 1/30).

Under light microscope, p-p38 and bcl-2 immunostaining was estimated as the percentage of neoplastic cells with nuclear and cytoplasmic reactivity, respectively, out of the total number of neoplastic cells counted. The same method was also applied for the assessment of cytoplasmic caspase- 8 immunostaining. However, because of a marked intratumoral heterogeneity of its expression, a score was given to sections based on the estimated percentage of immunopositive cells ( $1=\leq 25 \%, 2=26-50 \%$, $3=51-75 \%, 4=>75 \%)$ and on the intensity of the immunostaining $(1=$ no staining or weak, $2=$ moderate, $3=$ strong, $4=$ very strong). A final combined score of immunostaining was then calculated by adding both scores (weak $=$ score $2-3$, moderate $=$ score $4-6$, strong $=$ score $7-8$ ). The presence or absence of nuclear caspase- 8 staining was also assessed separately and scored as positive or negative ( $>10 \%$ or $\leq 10 \%$ positive cells out of the total number of neoplastic cells counted, respectively).

For statistical calculations, p-p38 expression and bcl-2 cytoplasmic expression as well as patients' age were treated as continuous variables. Cytoplasmic and nuclear 
caspase- 8 expression, tumor grade, pT-stage and patients' sex were analyzed as categorical variables. Associations among continuous variables were tested using Spearman's rank correlation coefficient whereas correlations among categorical variables using Chi-square or Fisher's exact test, as appropriate. The relationship between a continuous and a categorical variable was examined using Kruskal-Wallis ANOVA test or Mann-Whitney U-test, as appropriate.

Univariate survival analysis was performed using death due to disease as the endpoint. The effect of the various features on clinical outcome was assessed by plotting survival curves according to Kaplan-Meier method and comparing groups with the Log-rank test. Continuous variables were categorized on the basis of the median value rounded at its nearest $10 \%$. Caspase- 8 cytoplasmic expression was treated as a categorical variable (weak versus moderate/strong) as well as patients' gender. Grade and pT-stage were categorized in two groups (grade 1-2 versus grade 3-4 and pT1a-pT1b-pT2 versus pT3a-pT3b, respectively) due to the different biological behavior of these groups within ccRCCs [20,21]. All statistical calculations were completed using the statistical package SPSS, version 13.0, for Windows Software (SPSS, Chicago, Illinois, USA). Differences were considered statistically significant when p-value (two-sided) was $<0.05$.

\section{Results}

\section{Caspase-8, p-p38 and bcl-2 expression}

Caspase-8 cytoplasmic expression was detected in 27/27 cases [percentage of positive neoplastic cells: 15\%-90\% (mean value: 48.85\%)] [Fig. 1]. Thus, the cytoplasmic score was as follows: score $1(n=7$ cases), score $2(n=8)$, score $3(n=8)$, score $4(n=4)$. The intensity of cytoplasmic staining was as follows: score $1(\mathrm{n}=2)$, score $2(\mathrm{n}=$ $8)$, score $3(n=9)$, score $4(n=8)$. Therefore, the final caspase- 8 combined cytoplasmic score was: score 1 (weak immunostaining: $\mathrm{n}=5$ ), score 2 (moderate immunostaining: $n=13$ ), score 3 (strong immunostaining: $n=9$ ). Concerning nuclear immunoreaction, 18/27 cases were categorized as positive whereas the remaining cases were negative.

p-p38 was detected in 24/27 cases and was exclusively localized in the nuclei of malignant cells [percentage of positive neoplastic cells: $1 \%-85 \%$ (mean value: $19.59 \%$ )] [Fig. 1].

Bcl-2 cytoplasmic immunoreactivity was detected in 27/ 27 cases [percentage of positive neoplastic cells: 5\%-80\% (mean value: $45.19 \%$ )] [Fig. 2].

\section{Correlations among factors and Fuhrman's grade}

In our cohort, cytoplasmic caspase- 8 was negatively associated with grade in a statistically significant level [Fisher's Exact Test, $p=0.019]$. Nuclear caspase- 8 was not connected with grade [Fisher's Exact Test, $\mathrm{p}=0.424$ ].

p-p38 expression significantly increased from grade 1 through grade 4 tumors [Kruskal Wallis test, $\mathrm{p}=0.006$ ] [Fig. 3].

Bcl-2 expression was inversely associated with grade in a statistically significant level [Kruskal Wallis test, $\mathrm{p}=$ 0.041] [Fig. 4].

Finally, patients' age and gender were unrelated to grade, in our series [Kruskal Wallis Test, $\mathrm{p}=0.601$ and Fisher's Exact Test, $\mathrm{p}=0.845$, respectively].

\section{Correlations among caspase-8, p-p38, bcl-2 and pT-stage}

$\mathrm{Bcl}-2$ expression was inversely associated with pT-stage in the entire cohort [Kruskal Wallis test, $\mathrm{p}=0.026$ ].

p-p38 as well as cytoplasmic and nuclear caspase- 8 were not significantly associated with pT-stage in our group [Kruskal Wallis test $/ \mathrm{p}=0.604$, Fisher's Exact Test $/ \mathrm{p}=$ 0.605 and $\mathrm{p}=0.302$, respectively].

Patients' age and gender as well as grade were not statistically related to pT-stage [Kruskal Wallis Test/p $=0.714$, Fisher's Exact Test $/ \mathrm{p}=0.623$, Fisher's Exact Test $/ \mathrm{p}=0.101$, respectively].

\section{Correlations among caspase-8, p-p38, bcl-2, age and gender}

No statistically significant associations were detected among p-p38, caspase-8, bcl-2, patients' age and gender. However, high p-p38 expression tended to be associated with low immunopositivity for cytoplasmic caspase- 8 and high immunoreactivity for nuclear caspase-8 [Kruskal Wallis Test, $\mathrm{p}=0.202$ and $\mathrm{p}=0.523$, respectively]. p-p38, also, tended to be inversely related to bcl-2 expression [Spearman's rank correlation coefficient, $\mathrm{p}=0.433$ ].

\section{Survival analysis}

Univariate analysis demonstrated that higher cytoplasmic caspase-8 [Log Rank Test, weak versus moderate/strong, p $=0.037$ ] as well as lower grade [Log Rank Test, grade 1 and 2 versus grade 3 and $4, \mathrm{p}=0.019$ limplied a greater probability of survival, in the series of 27 patients [Fig. 5, Table 1].

The mean survival time of patients with high (moderate/ strong) cytoplasmic caspase-8 immunoreactivity was 72.77 months whereas the corresponding time for patients with weak caspase- 8 cytoplasmic immunoreactiv- 


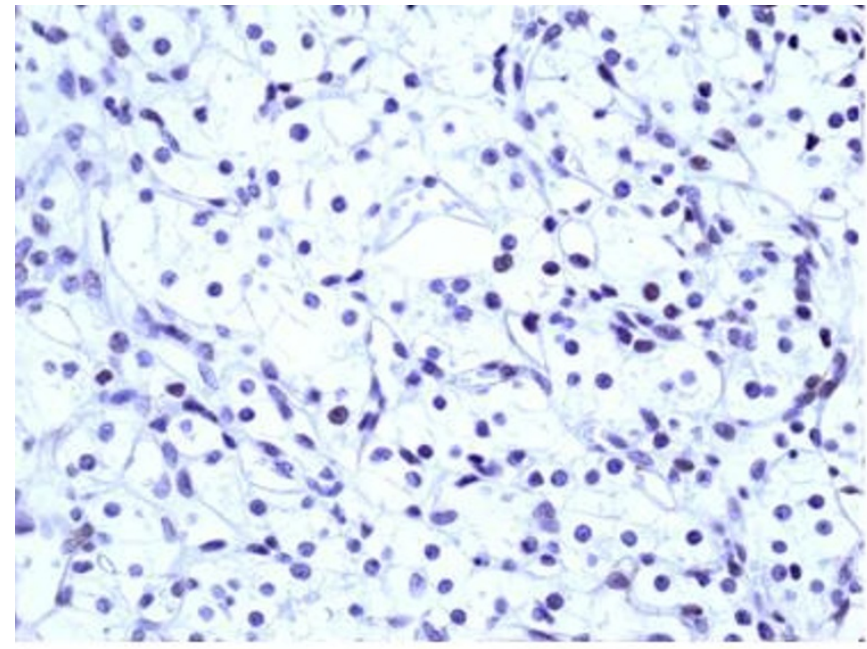

Fig. 1a

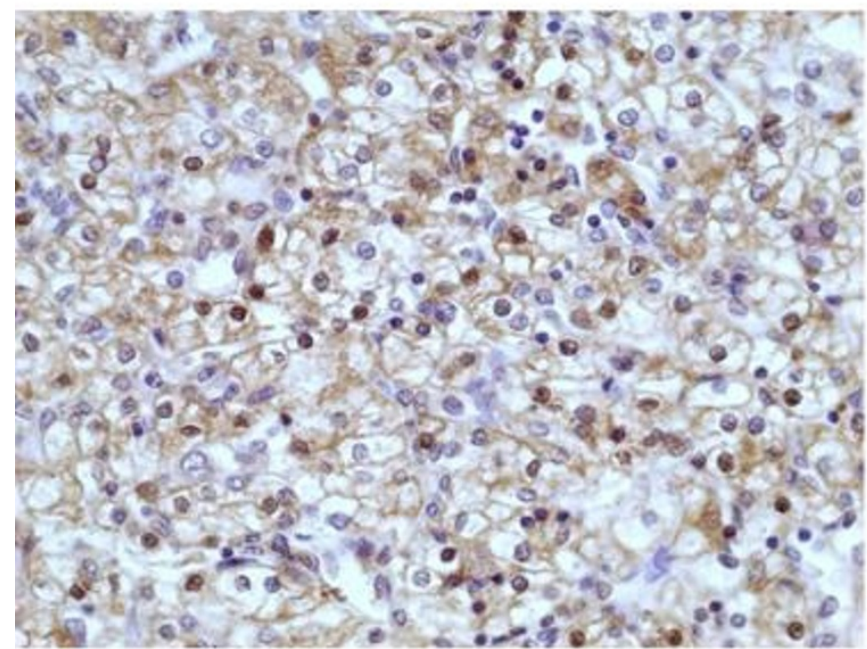

Fig. 1c

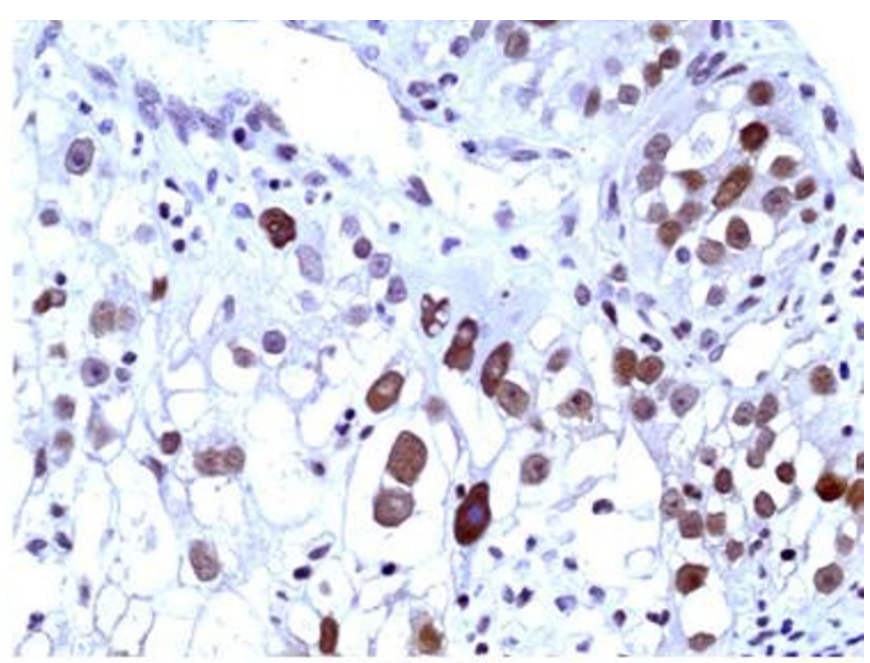

Fig. $1 \mathrm{~b}$

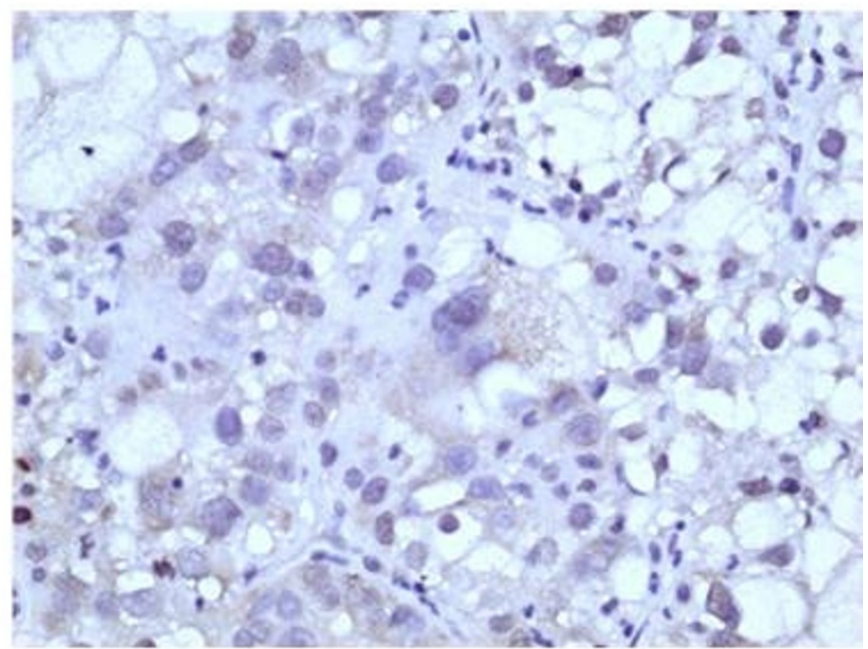

Fig. 1d

\section{Figure I}

p-p38 and caspase-8 expression in ccRCC. A): ( $p-p 38 \times 400)$. $p-p 38$ nuclear expression in few malignant cells in a grade I ccRCC. B): (p-p38 X400). High p-p38 nuclear expression from the majority of neoplastic cells in a grade $4 \mathrm{ccRCC}$. C): (caspase-8 X400). Strong cytoplasmic and nuclear expression of caspase-8 in a grade I ccRCC. D): (caspase-8 X400). Weak cytoplasmic as well as nuclear expression of caspase-8 in a grade 4 ccRCC. ccRCC: clear cell renal cell carcinoma.

ity was 52 months. The mean survival time of patients with low-grade tumors (grade 1 and 2) was 74.40 months while patients with high-grade neoplasms (grade 3 and 4) characterized by a mean survival of 59.86 months.

The remaining features (age, gender, pT-stage, p-p38, nuclear caspase-8, bcl-2) were not significantly related to patients' survival [Table 1].

\section{Discussion}

In the present study, we observed a significant negative association of cytoplasmic caspase- 8 with grade of
ccRCCs; cytoplasmic caspase-8 was highest in low-grade tumors, in contrast to previous studies in breast and lung carcinomas $[22,23]$. This negative relationship implies that caspase- 8 exerts an apoptotic action within low-grade ccRCCs, hence enhanced cytoplasmic distribution in grade 1 and 2 tumors. Our results seem to reflect the process in which malignant cells of high-grade specimens simultaneously display enhanced proliferative and decreased apoptotic activity $[5,24]$.

A significant association was noted between increased caspase- 8 cytoplasmic expression and better patients' sur- 


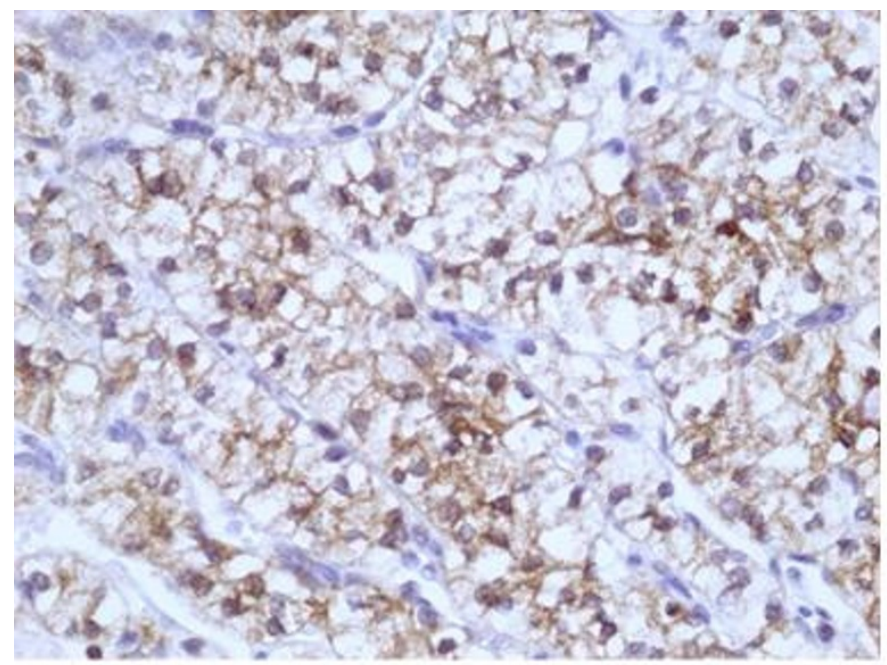

Fig. $2 \mathrm{a}$

Fig. $2 b$

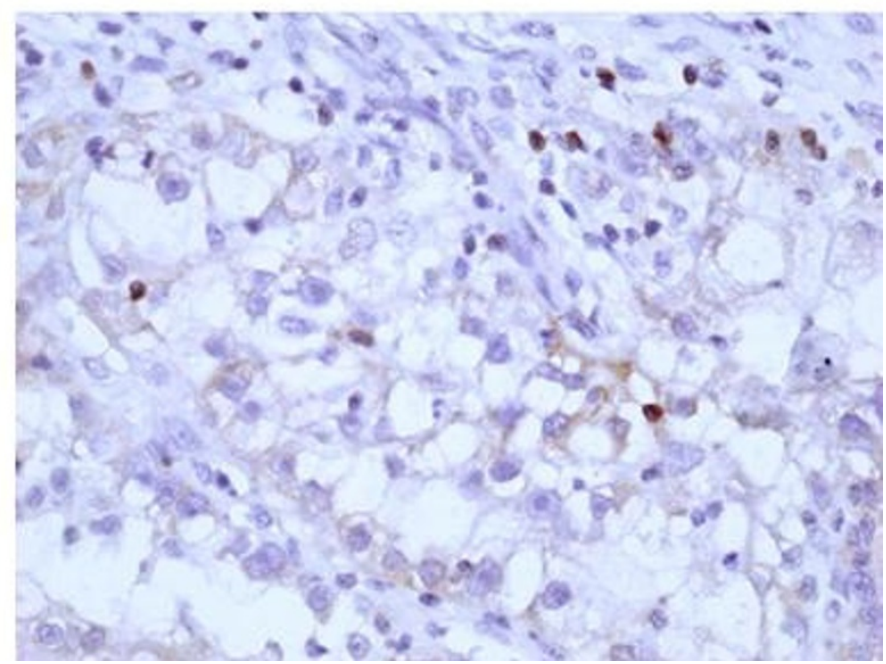

Figure 2

Bcl-2 expression in ccRCC. A): (Bcl-2 X400). Strong cytoplasmic expression of bcl-2 protein in a grade I ccRCC. B): (Bcl$2 \times 400$ ). Weak cytoplasmic expression of bcl-2 protein in a few malignant cells in a grade $3 \mathrm{ccRCC}$. Note also the positive inflammatory cells. ccRCC: clear cell renal cell carcinoma.

vival. This implication has never been reported in RCCs and is in agreement to recently published findings in papillary thyroid carcinomas [25].

p-p38 was detected in the majority of carcinomas localized in the nuclei of the malignant cells, in agreement with a previous study in ccRCCs [12]. Increased p-p38 expression was positively related to high grade ccRCCs, in line with studies performed in a variety of tumors $[26,27]$. To our knowledge, this is the first study in ccRCCs demonstrating this kind of association.
In contrast to published data regarding the apoptotic and anti-proliferative action of p-p38 in RCCs, our results suggest that p-p38 seems to exhibit a proliferative or antiapoptotic action, being increased in high-grade tumors [11]. This finding is also strengthened by the identification of decreased expression of the apoptotic cytoplasmic caspase- 8 with increasing the degree of malignancy within our group. Indeed, investigators have showed that bone morphogenetic protein 6 played its anti-apoptotic effect in breast cancer through activation of p38 pathway [28].

We additionally detected, in harmony with others, a significant inverse relationship between bcl-2 expression and 


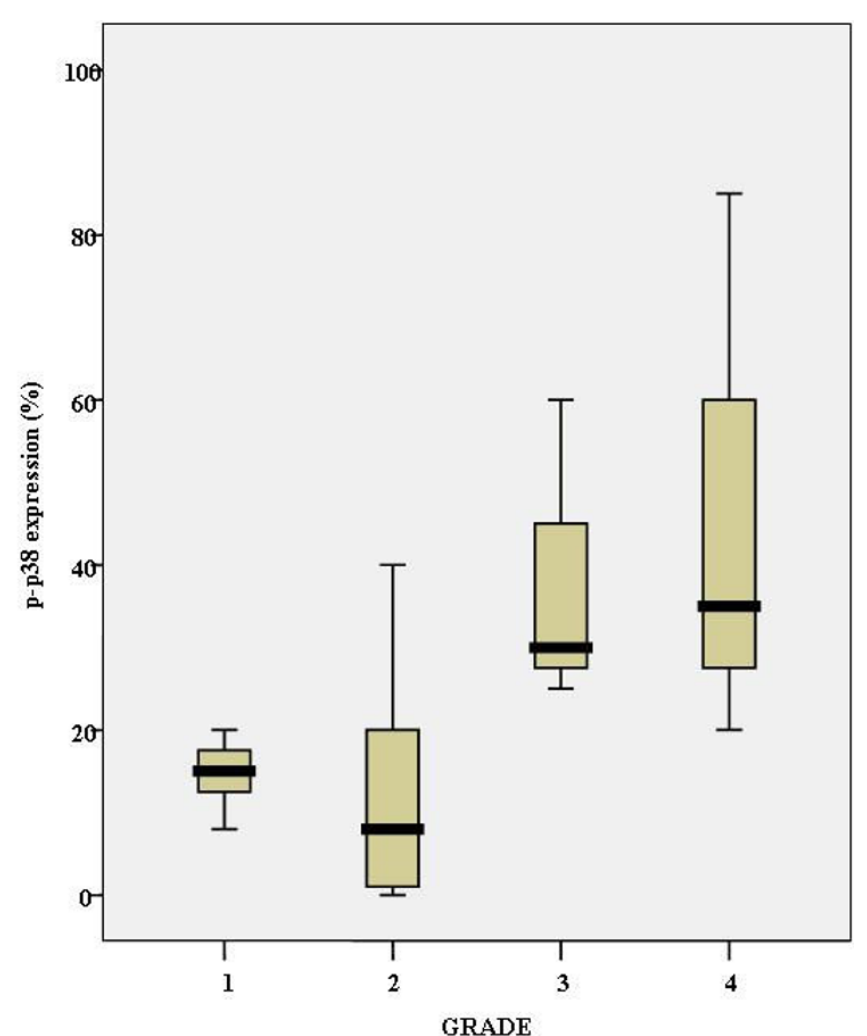

Figure 3

Schematic illustration of the relationship of p-p38 expression with Fuhrman's grade of 27 clear cell renal cell carcinomas. P-p38 expression increases with increasing grade in the entire cohort.

grade as well as pT-stage of RCCs [6,29]. A possible explanation to this might be based on the suggested antiproliferative role of bcl-2 protein [6,30]. This function, which seems to be distinct from its well-known antiapoptotic action, has been reported in various malignancies [31,32]. Therefore, it could be hypothesized that high expression of bcl-2 prevents cell proliferation, suppresses tumor growth and thereby is associated with a lower grade and pT-stage in RCCs, as previously stated [6].

Provided that a possible but not yet adequately studied mechanism, linking the p38 pathway with caspases activity has been previously implied [14,15], we attempted to correlate the expression of caspase- 8 and p-p38 in our series of RCCs. In this framework, researchers have suggested that caspase- 8 is essential for the activation of the p38 pathway through death receptors [33], while others pointed out that an enhanced activation of p38 kinase pathway may result in caspase- 8 activation and enhancement of apoptosis, based on experiments in a human astrocytoma cell line [34].

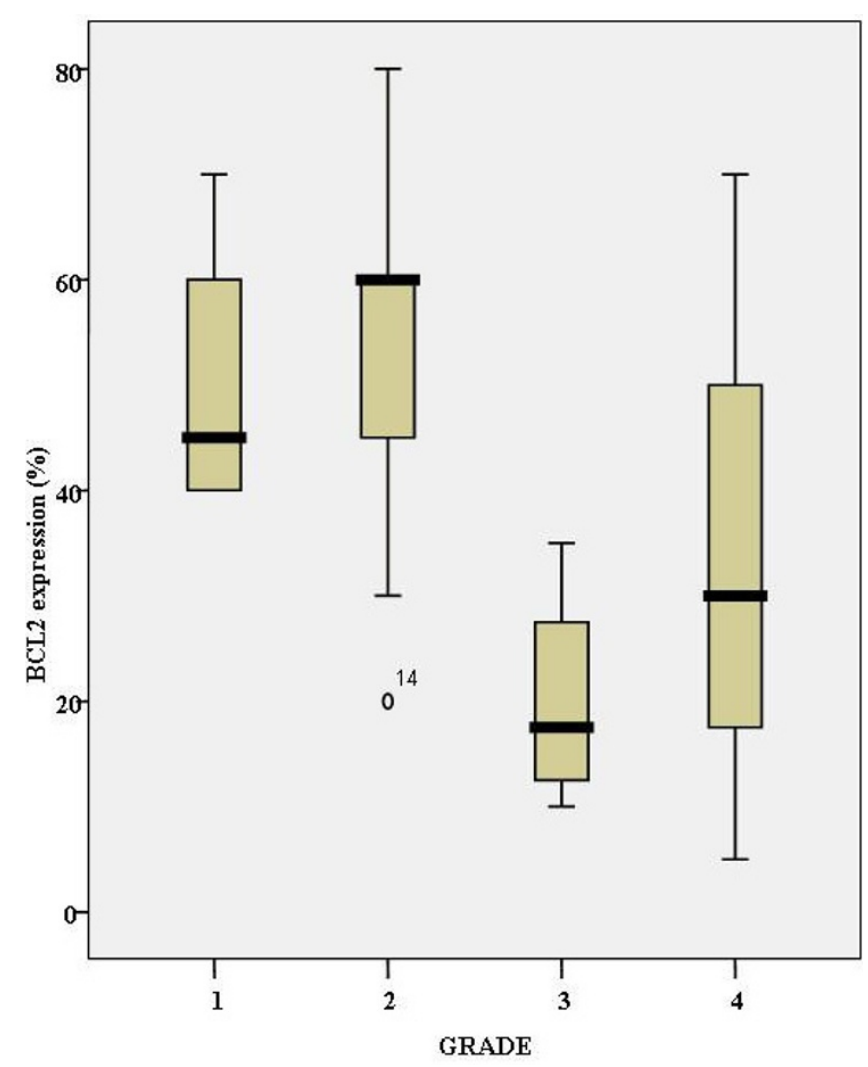

Figure 4

Schematic illustration of the relationship of bcl-2 expression with Fuhrman's grade of 27 clear cell renal cell carcinomas. Bcl-2 expression is inversely associated with grade in the entire cohort.

However, no statistically significant associations between cytoplasmic or nuclear caspase- 8 and p-p38 were revealed in our work, but only a suggestive negative and positive relationship, respectively. These findings backup our aforesaid hypothesis regarding the apoptotic action of cytoplasmic caspase- 8 and proliferative or anti-apoptotic role of p-p38 within our ccRCCs $[28,35]$. Moreover, we cannot exclude a potential inhibitory effect of p38 signaling upon caspase activity, as it has been previously reported [36]. The reason however, for the increased, though statistically insignificant, expression of nuclear caspase- 8 in parallel with high p-p38 immunoreactivity, is still to be determined.

As regards p-p38 and bcl-2 association, no strong linkage was identified in our study. However, p-p38 tended to be inversely related to bcl-2 expression. In this context, scientists have stressed that $\mathrm{p} 38$ can induce apoptosis via phosphorylation of bcl-2 [16,17]. Concerning RCCs, there are no related studies. According to our work, the negative, though loose, correlation found between p-p38 and bcl-2, constitutes an early indication of a probable antagonistic 

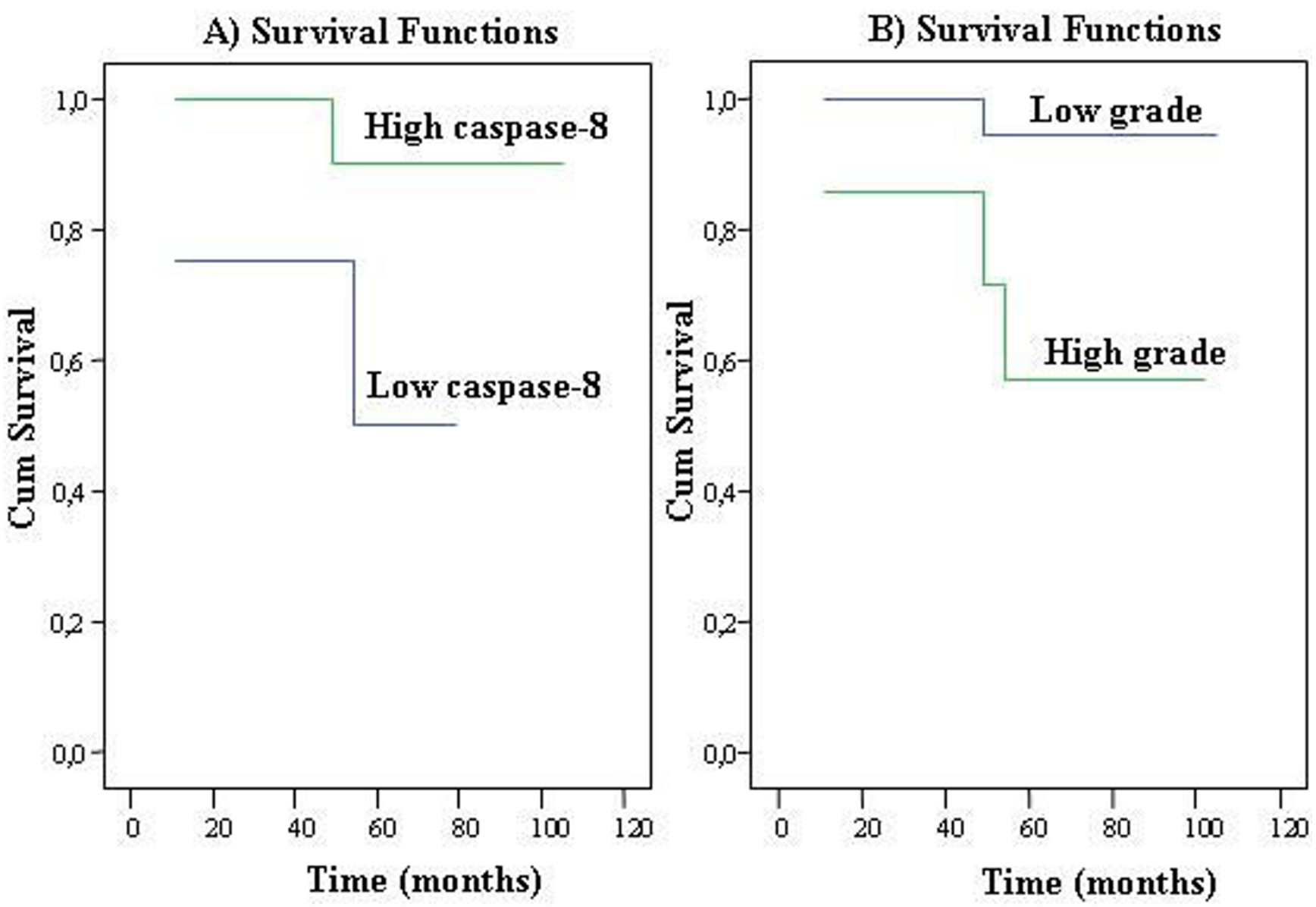

Figure 5

Kaplan-Meir survival curves in 27 patients with clear cell renal cell carcinomas according to: A) Cytoplasmic caspase-8 expression [green line indicates high expression (moderate/strong) while blue line low expression (weak)]. B) Fuhrman's grade [green line indicates high grade (3 and 4) while blue line low grade (I and 2)]. According to the statistical analysis, Fuhrman's grading is more significant as a prognosticator than caspase-8 cytoplasmic expression. Cum survival: probability of being a patient alive. Time (months): months since diagnosis.

interaction of these two factors within ccRCCs. The major question to be identified is whether this interaction concerns the proliferative, the apoptotic or both potential functions of these factors.

No statistically important correlation between bcl-2 and caspase- 8 was found. This was expected, since these substances act through different apoptotic pathways $[3,7]$. We also demonstrated that low-grade tumors (grade 1 and 2) depicted greater probability of survival in comparison to high-grade (grade 3 and 4), in accordance with previous data $[20,21]$. The absence of statistical significance regarding the association of pT-stage with prognosis could be attributed to the small cohort.

\section{Conclusion}

Conclusively, caspase- 8 pro-apoptotic function appears to prevail in low-grade ccRCCs and seems to be associated with a better clinical outcome. However, the specific role of cytoplasmic and nuclear caspase- 8 needs to be identified. Moreover, p-p38 seems to exert a proliferative rather than an apoptotic role within ccRCCs and is associated with an aggressive phenotype. Finally, the common antiapoptotic role of bcl-2 protein is extremely complicated within ccRCCs and appears to be influenced by other synergic and/or antagonistic pathways. In this regard, p38 MAPK signaling may be of fundamental importance. p38, caspase- 8 and bcl-2, though seemingly distinct components of three discrete signaling pathways implicated in cell growth and apoptosis, appear to be characterized, to some extent, by an interrelationship. p38 seems to bridge the above pathways and, in specific tissue substrates, reg- 
Table I: The association of studied features with prognosis (univariate survival analysis in the entire cohort)

\begin{tabular}{ll}
\hline Feature & P value (Log Rank Test) \\
\hline Phospho-p38 expression (<15 vs $\geq 15)^{*}$ & 0.443 \\
Cytoplasmic caspase-8 expression (weak vs moderate/strong) & $\mathbf{0 . 0 3 7}$ \\
Nuclear caspase-8 expression $(<10$ vs $\geq 10)$ & 0.257 \\
Bcl-2 expression $(<45 \text { vs } \geq 45)^{*}$ & 0.218 \\
Grade (grade I/2 vs grade 3/4) & $\mathbf{0 . 0 1 9}$ \\
PT-stage (stage PTIa/pTI b/pT2 vs PT3a/pT3b) & 0.712 \\
Age $(<54 \text { vs } \geq 54)^{*}$ & 0.273 \\
Gender (male vs female) & 0.660 \\
\hline
\end{tabular}

The association of studied features with prognosis was studied with the Log Rank Test. As shown in the table, grade and cytoplasmic caspase-8 expression are both prognosticators, and of these, Fuhrman's grade is more significant. Numbers in bold: statistically significant.

(*): median values.

ulates the balance between apoptotic cell death and proliferation. Prospective, larger, experiments are of utmost importance towards the elucidation of the exact clinical role and associations among caspase- $8, \mathrm{p}-\mathrm{p} 38$ and bcl-2 within the various subtypes as well as the different grade and stage categories of RCCs.

\section{Competing interests}

The authors declare that they have no competing interests.

\section{Authors' contributions}

VS Conception and design of study, writing of manuscript, histological diagnosis. MT Writing of manuscript, acquisition of clinical data, immunohistochemical staining. AS Writing of manuscript. CA Analysis and interpretation of statistical data. ES Writing of manuscript, drafting the manuscript. MA Analysis and interpretation of statistical data. HP Revising of manuscript. CB Revising and editing of manuscript, histological diagnosis. All authors read and approved the final manuscript.

\section{References}

I. Motzer RJ, Russo P: Systemic therapy for renal cell carcinoma. J Urol 2000, I 63(2):408-4I 7.

2. Kausch I, Jiang $H$, Thode $B$, et al.: Inhibition of bcl-2 enhances the efficacy of chemotherapy in renal cell carcinoma. Eur Urol 2005, 47(5):703-709.

3. Gross A, McDonnell JM, Korsmeyer SJ: BCL-2 family members and the mitochondria in apoptosis. Genes Dev 1999, I3(15): I899-1911.

4. Sejima T, Miyagawa I: Expression of bcl-2, p53 oncoprotein, and proliferating cell nuclear antigen in renal cell carcinoma. Eur Urol 1999, 35(3):242-248.

5. Zhang $X$, Takenaka I: Cell proliferation and apoptosis with BCL-2 expression in renal cell carcinoma. Urology 2000, 56(3):510-515.

6. Itoi T, Yamana K, Bilim V, et al.: Impact of frequent Bcl-2 expression on better prognosis in renal cell carcinoma patients. $\mathrm{Br}$ J Cancer 2004, 90(I):200-205.

7. Besnault-Mascard L, Leprince C, Auffredou MT, et al: Caspase-8 sumoylation is associated with nuclear localization. Oncogene 2005, 24(20):3268-3273.

8. Ramp U, Caliskan E, Mahotka C, et al:: Apoptosis induction in renal cell carcinoma by TRAIL and gamma-radiation is impaired by deficient caspase-9 cleavage. Br J Cancer 2003, 88( I I): |800-|807.
9. Jin X, Wu XX, Abdel-Muneem Nouh MA, et al: Enhancement of death receptor 4 mediated apoptosis and cytotoxicity in renal cell carcinoma cells by subtoxic concentrations of doxorubicin. J Urol 2007, I77(5): 1894-1899.

10. Heikaus S, Kempf T, Mahotka C, et al.: Caspase-8 and its inhibitors in RCCs in vivo: the prominent role of ARC. Apoptosis 2008, I 3(7):938-949.

II. Ambrose M, Ryan A, O'Sullivan GC, et al.: Induction of apoptosis in renal cell carcinoma by reactive oxygen species: involvement of extracellular signal-regulated kinase $1 / 2$, p38delta/ gamma, cyclooxygenase-2 down-regulation, and translocation of apoptosis-inducing factor. Mol Pharmacol 2006, 69(6): $1879-1890$.

12. Huang D, Ding Y, Luo WM, et al.: Inhibition of MAPK kinase signaling pathways suppressed renal cell carcinoma growth and angiogenesis in vivo. Cancer Res 2008, 68(I):8I-88.

13. Romanenko A, Morell-Quadreny L, Lopez-Guerrero JA, et al.: The INK4a/ARF locus: role in cell cycle control for renal cell epithelial tumor growth after the Chernobyl accident. Virchows Arch 2004, 445(3):298-304.

14. Cahill MA, Peter ME, Kischkel FC, et al.: CD95 (APO-I/Fas) induces activation of SAP kinases downstream of ICE-like proteases. Oncogene 1996, I3(10):2087-2096.

15. Ono K, Han J: The p38 signal transduction pathway: activation and function. Cell Signal 2000, I 2(I): I-I3.

16. Torcia M, De Chiara G, Nencioni L, et al.: Nerve growth factor inhibits apoptosis in memory $B$ lymphocytes via inactivation of p38 MAPK, prevention of $\mathrm{Bcl}-2$ phosphorylation, and cytochrome c release. J Biol Chem 200I, 276(42):39027-39036.

17. Bu SZ, Huang Q, Jiang YM, et al.: p38 Mitogen-activated protein kinases is required for counteraction of 2-methoxyestradiol to estradiol-stimulated cell proliferation and induction of apoptosis in ovarian carcinoma cells via phosphorylation Bcl2. Apoptosis 2006, I I (3):4 I3-425.

18. Fuhrman SA, Lasky LC, Limas C: Prognostic significance of morphologic parameters in renal cell carcinoma. Am J Surg Pathol 1982, 6(7):655-663.

19. Sobin LH, Wittekind CH: TNM Classification of Malignant Tumors 6th edition. New York: Wiley-Liss; 2002.

20. Tsui KH, Shvarts O, Smith RB, et al.: Prognostic indicators for renal cell carcinoma: a multivariate analysis of 643 patients using the revised 1997 TNM staging criteria. J Urol 2000, I 63(4): 1090-1095.

21. Zubac DP, Bostad L, Gestblom C, et al.: Renal cell carcinoma: a clinicopathological follow-up study after radical nephrectomy. Scand J Urol Nephrol 2007, 4I(3): I 91 - 197.

22. Vakkala M, Pääkkö $P$, Soini $Y$ : Expression of caspases 3, 6 and 8 is increased in parallel with apoptosis and histological aggressiveness of the breast lesion. Br J Cancer 1999, 8 I (4):592-599.

23. Shen J, Behrens C, Wistuba II, et al.: Identification and validation of differences in protein levels in normal, premalignant, and malignant lung cells and tissues using high-throughput Western Array and immunohistochemistry. Cancer Res 2006, 66(23): I | |94-I I206. 
24. Kikuchi $Y$, Dinjens WN, Bosman FT: Proliferation and apoptosis in proliferative lesions of the colon and rectum. Virchows Arch 1997, 43 I(2): I II-II 7.

25. Mitsiades CS, Poulaki V, Fanourakis G, et al.: Fas signaling in thyroid carcinomas is diverted from apoptosis to proliferation. Clin Cancer Res 2006, I 2( I 2):3705-37I2.

26. Miki H, Yamada H, Mitamura K: Involvement of p38 MAP kinase in apoptotic and proliferative alteration in human colorectal cancers. Anticancer Res 1999, 19(6B):5283-5291.

27. Demuth T, Reavie LB, Rennert JL, et al.: MAP-ing glioma invasion: mitogen-activated protein kinase kinase 3 and p38 drive glioma invasion and progression and predict patient survival. Mol Cancer Ther 2007, 6(4): $1212-1222$.

28. Du J, Yang S, Wang Z, et al.: Bone morphogenetic protein 6 inhibit stress-induced breast cancer cells apoptosis via both Smad and p38 pathways. J Cell Biochem 2008, I03(5): I 584- 1597.

29. Lipponen P, Eskelinen M, Syrjänen K: Expression of tumour-suppressor gene $\mathbf{R b}$, apoptosis-suppressing protein $\mathrm{Bcl}-2$ and $\mathrm{c}-$ Myc have no independent prognostic value in renal adenocarcinoma. Br J Cancer 1995, 7 I (4):863-867.

30. Pierce $\mathrm{RH}$, Vail ME, Ralph L, et al.: Bcl-2 expression inhibits liver carcinogenesis and delays the development of proliferating foci. Am J Pathol 2002, 160(5): 1555-1560.

3।. Bozzetti C, Nizzoli R, Naldi N, et al.: Bcl-2 expression on fine-needle aspirates from primary breast carcinoma: correlation with other biologic factors. Cancer 1999, 87(4):224-230.

32. Ishida $\mathrm{H}$, Irie $\mathrm{K}$, Itoh $\mathrm{T}$, et al:: The prognostic significance of $\mathrm{p} 53$ and $\mathrm{bcl}-2$ expression in lung adenocarcinoma and its correlation with Ki-67 growth fraction. Cancer 1997, 80(6): I034- 1045.

33. Juo P, Kuo CJ, Yuan J, et al:: Essential requirement for caspase8/FLICE in the initiation of the Fas-induced apoptotic cascade. Curr Biol 1998, 8(18): I00I-1008.

34. Ohtsuka T, Zhou T: Bisindolylmaleimide VIII enhances DR5mediated apoptosis through the MKK4/JNK/p38 kinase and the mitochondrial pathways. J Biol Chem 2002, 277(32):29294-29303.

35. Abdollahi T, Robertson NM, Abdollahi A, et al:: Inhibition of TRAIL-induced apoptosis by IL-8 is mediated by the p38MAPK pathway in OVCAR3 cells. Apoptosis 2005, I 0(6): 1383-1393.

36. Alvarado-Kristensson M, Melander F, Leandersson K, et al.: p38MAPK signals survival by phosphorylation of caspase- 8 and caspase-3 in human neutrophils. J Exp Med 2004, 199(4):449-458

Publish with Biomed Central and every scientist can read your work free of charge

"BioMed Central will be the most significant development for disseminating the results of biomedical research in our lifetime. "

Sir Paul Nurse, Cancer Research UK

Your research papers will be:

- available free of charge to the entire biomedical community

- peer reviewed and published immediately upon acceptance

- cited in PubMed and archived on PubMed Central

- yours - you keep the copyright
BioMedcentral 